\title{
Bifurcating electric wires
}

\author{
E.W.C. VAN GROESEN
}

Mathematical Institute, University of Nijmegen, The Netherlands

Summary. The stationary states of a string through which an electric current is sent and which is placed in an axial magnetic field, are investigated. Using methods of constrained variational principles, it is shown that, in case the string is inextensible, only those stationary states which have least total potential energy are stable.

\section{Introduction}

The study of the dynamical behaviour of strings and bars, although interesting in itself, is especially well suited to demonstrate various methods of mathematical physics. One of the best examples I know of that can lend support to this statement is the paper 'On the dynamics of strings' by Broer [1]. In that paper it is shown how the full set of nonlinear equations for inextensible, and for elastic, strings can be obtained from an action principle, thereby introducing, for the inextensible case, a Lagrangian multiplier (which can be interpreted as the stress) to take account of the constant length condition. One of the main lessons that can be learned from Broer's paper, is that the usual treatment of the linear theory of inextensible strings can only be understood after a thorough investigation of the nonlinear equations. Together with a comparable paper on the dynamics of bars [2], also announced as being '... mainly of a didactical character', this paper has been the starting point for several students to work on topics from the theory of strings and bars (see also [3]). In fact, the underlying paper has grown out of my master thesis [4], which was prepared several years ago under the supervision of Broer. Although time has not left unaltered the presentation and treatment of the original subject, several ideas of [4] are also present in this paper.

In this paper it is shown that the equations for time-independent solutions of a simple electromechanical system, which consists of an electric wire placed in a magnetic field and satisfying suitable boundary conditions, provide us with a model that shows the phenomenon of bifurcation. As the stationary solutions can be described explicitly in terms of elementary functions, this model is even simpler than the Euler-buckling of a rod, the well-known classical example of a bifurcation problem.

In Section 2 we describe the system, and determine the stationary states, when it is assumed that the electric wire is inextensible; this is the model originally proposed by Broer. In Section 5 we consider the same electromechanical system, but now with an elastic wire. As there is freedom in choosing the elastic potential energy function, this last system can describe various, rather different, bifurcation problems with the same ease. For the inextensible string model, stability of the stationary states will be investigated 
by using constrained variational principles and the multiplier rule in a consequent way. The stationary states are the stationary points of the constrained total potential energy functional. In Section 4 it is shown how (the lack of) extremality properties of such a constrained stationary point implies the (un-) stability of it as a stationary state of the constrained dynamical system, in much the same way as is known for dynamical systems without constraints. The investigation of such extremality properties of these constrained stationary points is therefore the subject of Section 3 .

\section{The inextensible string model}

The first electromechanical system to be considered consists of an homogeneous, completely flexible string of constant length, normalized to be $2 \pi$, and constant normalized mass density 1 per unit length. One of the ends of the string is fixed at the origin of a Cartesian coordinate system, while the other one can move over the positive $x$-axis subject to a constant external force $\bar{\sigma}_{0} \mathrm{e}$, where $\mathrm{e}=(1,0,0)$ and $\bar{\sigma}_{0}>0$.

An electric current of constant magnitude $J$ is sent through the string, and this configuration is placed in a constant external magnetic field $B$; only positive values of the parameter $\lambda:=J B$ will be considered. The position of a point at the string is described with the vector function $\mathbf{r}=\mathbf{r}(s, t)=(x(s, t)$, $y(x, t), z(s, t))$, where $t$ is the time and $s$ is the arc length measured from the origin, $0 \leqslant s \leqslant 2 \pi$. By definition of arc length, the tangent vector $\mathbf{r}_{\boldsymbol{s}}$ has unit length:

$$
\mathbf{r}_{s} \cdot \mathbf{r}_{s}=1 \text {. }
$$

The equations of motion will be derived with the aid of a Lagrangian, which is taken to be the difference of kinetic and potential energy:

$$
L\left(\mathbf{r}, \mathbf{r}_{t}\right)=K\left(\mathbf{r}_{t}\right)-P_{\lambda}(\mathbf{r})
$$

where

$$
K\left(\mathbf{r}_{t}\right)=\int_{0}^{2 \pi} \frac{1}{2} \mathbf{r}_{t} \cdot \mathbf{r}_{t} \mathrm{~d} s
$$

and

$$
P_{\lambda}(\mathbf{r})=\int_{0}^{2 \pi} \frac{1}{2} \lambda \mathrm{r}_{s} \cdot(\mathbf{r} \times \mathbf{e}) \mathrm{d} s+\bar{\sigma}_{0}(2 \pi-\mathrm{r}(2 \pi, t) \cdot \mathrm{e})
$$

According to Hamilton's principle we look for stationary points of the action functional $\int_{t_{2}}^{t_{2}} L\left(\mathbf{r}, \mathbf{r}_{t}\right) \mathrm{d} t$ on the set of functions $\mathbf{r}(s, t)$ that satisfy, apart from initial and final values at $t=t_{1}$ and $t=t_{2}$, the condition (2.1) and the boundary condition $\mathbf{r}(0, t)=\mathbf{0}, \mathbf{r}(2 \pi, t) \times \mathbf{e}=\mathbf{0}$. As the constraint (2.1) satisfies all the conditions for the (Lagrange) multiplier rule to be applicable 
(see van Groesen [5, Section 1.7.1]) it follows that stationary points satisfy for some multiplier $\sigma=\sigma(s, t)$ the following set of equations:

$$
\begin{aligned}
& \mathbf{r}_{t t}=\left(\sigma \mathbf{r}_{s}\right)_{s}+\lambda \mathbf{r}_{s} \times \mathrm{e} \\
& \mathbf{r}_{s} \cdot \mathbf{r}_{s}=1 \\
& \sigma(2 \pi, t) \mathbf{r}_{s}(2 \pi, t) \cdot \mathrm{e}=\bar{\sigma}_{0} \\
& \mathrm{r}(0, t)=0, \quad \mathbf{r}(2 \pi, t) \times \mathbf{e}=\mathbf{0}
\end{aligned}
$$

Physically, the multiplier $\sigma(s, t)$ can be interpreted as the stress of the string, and the transversality condition (2.6) as a balance of forces at the endpoint $s=2 \pi$.

Time independent solutions of these equations have constant stress, as is seen by taking the inner product of equation (2.5) with the tangent vector $\mathbf{r}_{s}$. With this observation the stationary states are easily found: For any $\lambda>0$ the undeflected string configuration:

$$
\mathbf{r}_{0}=(s, 0,0)
$$

is a solution with stress $\sigma_{0}=\bar{\sigma}_{0}$.

Furthermore, for $n \in \mathbb{N}$, set $\lambda_{n}:=n \bar{\sigma}_{0}$, and let $R(\psi)$ be the rotation over an angle $\psi$ about the $x$-axis. Then for $n \geqslant 1$, and $\lambda>\lambda_{n}$ there are invariant sets $M_{n}(X)$ of continua of stationary states:

$$
\begin{aligned}
M_{n}(\lambda)= & \left\{\mathbf{r}_{n}=R(\psi) \hat{\mathbf{r}}_{n} \mid \hat{\mathbf{r}}_{n}=\right. \\
& \left(\cos \theta(\lambda) \cdot s \frac{1}{n} \sin \theta(\lambda) \sin n s, \frac{1}{n} \sin \theta(\lambda) \cdot(1-\cos n s)\right) \\
& \left.\cos \theta(\lambda)=\lambda_{n} / \lambda, 0 \leqslant \psi<2 \pi\right\}
\end{aligned}
$$

and for $\mathbf{r}_{n} \in M_{n}(\lambda)$ the stress is given by $\sigma_{n}(\lambda)=\lambda / n$. Note that $\mathbf{r}_{n}$ is a circle screw lying on a cylinder of radius

$$
R_{n}(\lambda)=\frac{1}{n} \sin \theta(\lambda)=\left\{\left(\frac{1}{n}\right)^{2}-\left(\frac{\bar{\sigma}_{0}}{\lambda}\right)^{2}\right\}^{1 / 2} .
$$

The total potential energy of the stationary states is given by

$$
\begin{aligned}
& P_{\lambda}^{0}:=P_{\lambda}\left(\mathbf{r}_{0}\right)=0, \\
& P_{\lambda}^{n}:=P_{\lambda}\left(\mathbf{r}_{n}\right)=-\bar{\sigma}_{0} \pi\left(\frac{\lambda}{\lambda_{n}}-1\right) \cdot\left(1-\frac{\lambda_{n}}{\lambda}\right)
\end{aligned}
$$

for all $\mathbf{r}_{n} \in M_{n}(\lambda), \quad n \geqslant 1, \quad \lambda>\lambda_{n}$.

Note that these energy levels are ordered in the following way:

$$
P_{\lambda}^{1}<P_{\lambda}^{2}<\ldots<P_{\lambda}^{n}<P_{\lambda}^{0}=0 \text { for } \lambda>\lambda_{n} .
$$


This implies that amongst the stationary states, $\mathbf{r}_{0}$ is the one which has the least value of the potential energy for $0<\lambda\left\langle\lambda_{1}\right.$, while for $\left.\lambda\right\rangle \lambda_{1}$ this property is taken over by the elements of $M_{1}(\lambda)$. Our aim is to show that the stationary states with least potential energy are stable solutions (in the sense of Lypunov) of the evolution equations, and that the other stationary states are unstable. To that end we can use properties of the potential energy functional on the set of functions which satisfy the constraint (2.1) by a slight modification of methods that are known for dynamical systems without constraints. Therefore, we shall first concentrate our investigations on the potential energy functional $P_{\lambda}$.

\section{The potential energy functional}

In this section the dependence on time of the functions is not essential and will be omitted. On the space of vector functions $\Sigma$ :

$$
\Sigma:=\{\mathbf{r}=\mathbf{r}(s) \mid \mathbf{r}(0)=0, \mathbf{r}(2 \pi) \times \mathbf{e}=\mathbf{0}\},
$$

we define the (first Sobolev) inner product $(,)_{1}$ and corresponding norm \|\|$_{1}$ in the standard way:

$$
\begin{aligned}
& \left(\boldsymbol{\xi}_{1}, \boldsymbol{\xi}_{2}\right)_{1}:=\left\langle\left(\boldsymbol{\xi}_{1}\right)_{s},\left(\boldsymbol{\xi}_{2}\right)_{s}\right\rangle+\left\langle\boldsymbol{\xi}_{1}, \boldsymbol{\xi}_{2}\right\rangle, \\
& \|\boldsymbol{\xi}\|_{1}:=\left\{\left\|\boldsymbol{\xi}_{s}\right\|^{2}+\|\boldsymbol{\xi}\|^{2}\right\}^{1 / 2},
\end{aligned}
$$

where $\langle$,$\rangle and \|\|$ are the usual $L_{2}$-inner product and $L_{2}$-norm respectively. As a consequence of the Poincaré-Friedrich inequality, the norm \|\|$_{1}$ is equivalent to the norm $\boldsymbol{\xi} \rightarrow\left\|\boldsymbol{\xi}_{s}\right\|$ on the space $\Sigma$.

For the following we need an auxiliary lemma. Therefore, introduce for $\mu>0$ the quadratic functional $Q_{\mu}$ :

$$
Q_{\mu}(\boldsymbol{\xi}):=\int_{0}^{2 \pi}\left\{\boldsymbol{\xi}_{s} \cdot \boldsymbol{\xi}_{s}+\mu \boldsymbol{\xi}_{s} \cdot(\boldsymbol{\xi} \times \mathbf{e})\right\} \mathrm{d} s, \quad \xi \in \Sigma
$$

This functional satisfies for $\mu>0$ the estimates

$$
\left\|\boldsymbol{\xi}_{s}\right\|^{2}-\mu\|\boldsymbol{\xi}\| \cdot\left\|\boldsymbol{\xi}_{s}\right\| \leqslant Q_{\mu}(\boldsymbol{\xi}) \leqslant(1+\mu)\|\boldsymbol{\xi}\|_{1}^{2} \quad \forall \xi \in \Sigma .
$$

Consequently, the eigenvalues of the eigenvalue problem associated with this functional are bounded from below: the lowest eigenvalue $\Lambda_{\mu}$ is finite:

$$
\Lambda_{\mu}:=\min \left\{Q_{\mu}(\boldsymbol{\xi}) \mid \boldsymbol{\xi} \in \Sigma ;\|\boldsymbol{\xi}\|^{2}=1\right\}>-\infty .
$$

Solving the eigenvalue problem for $Q_{\mu}$ explicitly, the following results are immediate:

\section{Lemma}

(i) For $0<\mu<1, \Lambda_{\mu}>0$; in particular, there exists a constant $c_{1}>0$ such that 


$$
Q_{\mu}(\boldsymbol{\xi}) \geqslant c_{1}\|\boldsymbol{\xi}\|_{1}^{2} \quad \forall \boldsymbol{\xi} \in \Sigma
$$

(ii) For $\mu=1, \Lambda_{1}=0$; in particular,

$$
\begin{aligned}
Q_{1}(\hat{\xi})= & 0 \quad \text { for } \\
\hat{\boldsymbol{\xi}} \in S= & \{a(0, \sin (s+\delta)-\sin \delta, \cos \delta-\cos (s+\delta)) \mid a \in \mathbb{R}, \\
& \delta \in[0,2)\},
\end{aligned}
$$

and there exists a constant $c_{2}>0$ such that

$$
\begin{gathered}
Q_{1}(\boldsymbol{\xi}) \geqslant c_{2}\|\boldsymbol{\xi}\|_{1}^{2} \quad \text { for all } \quad \boldsymbol{\xi} \in \Sigma \text { with } \quad(\boldsymbol{\xi}, \hat{\boldsymbol{\xi}})_{1}=0 \\
\forall \hat{\boldsymbol{\xi}} \in S .
\end{gathered}
$$

(iii) For $\mu>1, \Lambda_{\mu}<0$.

Let us now consider the potential energy functional $P_{\lambda}$ :

$$
P_{\lambda}(\mathbf{r}):=\int_{0}^{2 \pi} \lambda \mathbf{r}_{s} \cdot(\mathbf{r} \times \mathbf{e}) d s+\bar{\sigma}_{0}(2 \pi-\mathbf{r}(2 \pi) \cdot \mathbf{e})
$$

on the set of functions which satisfy the constraint (2.1) and the boundary conditions:

$$
\mathscr{M}:=\left\{\mathbf{r} \in \Sigma \mid \mathbf{r}_{s} \cdot \mathbf{r}_{s}=1\right\} .
$$

Clearly, stationary points of the functional $P_{\lambda}$ on $\mathscr{M}$ are time-independent solutions of the dynamical equations (2.5)-(2.7) and vice versa.

In the next section it will be shown that the stability of such a timeindependent solution is determined by its 'character' as a stationary point of the functional $P_{\lambda}$ on $\mathscr{M}$ (i.e. whether it is a minimal- or a saddle-point of $P_{\lambda}$ on $\mathscr{M}$ ). These properties of the stationary points are the subject of the following four theorems.

\section{Theorem 1}

For $0<\lambda<\lambda_{1}$ the functional $P_{\lambda}$ attains its infimum on $\mathscr{M}$ at $\mathbf{r}=\mathbf{r}_{0}$; moreover, $P_{\lambda}$ is non-degenerate at $\mathbf{r}_{0}$ : there exist constants $c>0$ and $\epsilon>0$ such that

$$
\begin{aligned}
& P_{\lambda}(\mathbf{r})-P_{\lambda}^{0} \geqslant c\left\|\mathbf{r}-\mathbf{r}_{0}\right\|_{1}^{2} \quad \text { for all } \quad \mathbf{r} \in \mathscr{M} \\
& \text { with }\left\|\mathbf{r}-\mathbf{r}_{0}\right\|_{1}<\epsilon .
\end{aligned}
$$

Proof: First observe that $P_{\lambda}$ is bounded from below on $\mathscr{M}$; using standard Hilbert space methods it can then be shown that the infimum is attained at some $\hat{\mathbf{r}} \in \mathscr{M}$. Then $\hat{\mathbf{r}}$ must be a stationary point of $P_{\lambda}$ on $\mathscr{M}$, and hence $\hat{\mathbf{r}}$ is the unique stationary point $\mathbf{r}_{0}$. In order to prove the estimate (3.8), consider the second variation of the functional $P_{\lambda}$ on $\mathscr{M}$ at $\mathbf{r}=\mathbf{r}_{0}$ : 


$$
J_{\lambda}^{0}(\xi)=\int_{0}^{2 \pi}\left\{\frac{1}{2} \bar{\sigma}_{0} \xi_{s} \cdot \xi_{s}+\frac{1}{2} \lambda \xi_{s} \cdot(\boldsymbol{\xi} \times \mathbf{e})\right\} \mathrm{d} s,
$$

for

$$
\boldsymbol{\xi} \in T \mathscr{M}\left(\mathbf{r}_{0}\right)=\left\{\boldsymbol{\xi} \in \Sigma \mid \boldsymbol{\xi}_{\mathrm{s}} \cdot \mathrm{e}=0\right\} .
$$

Here $T \mathscr{M}\left(\mathbf{r}_{0}\right)$ is the tangent space of $\mathscr{M}$ at $\mathbf{r}_{0}$, which consists of those elements $\boldsymbol{\xi} \in \Sigma$ for which $\mathbf{r}_{0}+\boldsymbol{\xi}$ satisfies the constraint $\mathbf{r}_{s} \cdot \mathbf{r}_{s}=1$ in first order. According to the lemma, part (i), the functional $J_{\lambda}^{0}$ satisfies

$$
J^{0}(\boldsymbol{\xi}) \geqslant \frac{1}{2} \bar{\sigma}_{0} c_{1}\|\xi\|_{1}^{2} \quad \forall \xi \in T \mathscr{H}\left(\mathbf{r}_{0}\right) .
$$

As is well known, (3.11) implies the result (3.8) (see e.g. Ioffe and Tihomirov [6, Section 7.2.2], van Groesen [5, Theorem 1.5.2]).

\section{Theorem 2}

For $\lambda>\lambda_{1}$ the functional $P_{\lambda}$ has not a local minimum on $\mathscr{M}$ at $\mathbf{r}=\mathbf{r}_{0}$ : the difference $P_{\lambda}(\mathbf{r})-P_{\lambda}^{0}$ is not sign definite on $\mathscr{M}$.

Proof: Again we consider the second variation $J_{\lambda}^{0}$, given by (3.9), on the tangent space $T \mathscr{M}\left(\mathrm{r}_{0}\right)$. According to the lemma, part (iii), $J_{\lambda}^{0}$ is not sign definite on $T \mathscr{H}\left(\mathrm{r}_{0}\right)$ : in fact, the lowest eigenvalue $\Gamma_{\lambda}^{0^{\circ}}$ corresponding to the functional $J_{\lambda}^{0}$ on $T \mathscr{M}\left(\mathbf{r}_{0}\right)$ :

$$
\Gamma_{\lambda}^{0}:=\min \left\{J_{\lambda}^{0}(\boldsymbol{\xi}) \mid \boldsymbol{\xi} \in T \mathscr{M}\left(\mathbf{r}_{0}\right):\|\xi\|^{2}=1\right\}
$$

is given by $\Gamma_{\lambda}^{0}=\frac{1}{2} \bar{\sigma}_{0} \cdot \Lambda_{\left(\lambda \lambda_{1}\right)}<0$. This property of the second variation on the tangent space implies the contents of the theorem (see e.g. Ioffe and Tihomirov [6, Section 7.2.1], van Groesen [5, Theorem 1.5.1]).

Next we shall show that any stationary state $\mathrm{r}_{1} \in M_{1}(\lambda)$ minimizes $P_{\lambda}$ on $\mathscr{M}$ for $\lambda>\lambda_{1}$. In order to be able to obtain an estimate for the difference $P_{\lambda}(\mathbf{r})-P_{\lambda}^{1}$ we need to introduce the distance $d\left(\mathbf{r}, M_{1}(\lambda)\right)$ of an element $\mathrm{r} \in \Sigma$ to the invariant set $M_{1}(\lambda)$ in the following way:

$$
\begin{aligned}
d\left(\mathbf{r}, M_{1}(\lambda)\right) & :=\min \left\{\left\|\mathbf{r}-\mathbf{r}_{1}\right\|_{1} \mid \mathbf{r}_{1} \in M_{1}(\lambda)\right\}= \\
& =\min \left\{\left\|\mathbf{r}-R(\psi) \hat{\mathbf{r}}_{1}\right\|_{1} \mid 0 \leqslant \psi<2 \pi\right\} .
\end{aligned}
$$

Consequently, for any $\mathbf{r} \in \Sigma$ there exist unique elements $\mathbf{r}_{1}$ and $\boldsymbol{\xi} \in \Sigma$ such that

$$
\mathbf{r}=\mathbf{r}_{1}+\boldsymbol{\xi}, \quad \text { with } \quad \mathbf{r}_{1}=R(\psi) \hat{\mathbf{r}}_{1}, \quad \text { and } \quad\left(\boldsymbol{\xi}, \dot{R}(\psi) \hat{\mathbf{r}}_{1}\right)_{1}=0 \text {, }
$$

where $\dot{R}(\psi):=(\mathrm{d} / \mathrm{d} \psi) R(\psi)$; then $d\left(\mathbf{r}, M_{1}(\lambda)\right)=\|\xi\|_{1}$.

\section{Theorem 3}

For $\lambda>\lambda_{1}$ the functional $P_{\lambda}$ attains its infimum on $\mathscr{M}$ at the elements of 
the invariant set $M_{1}(\lambda)$; moreover, there exist constants $c>0$ and $\epsilon>0$ such that

$$
\begin{aligned}
& P_{\lambda}(\mathbf{r})-P_{\lambda}^{1} \geqslant c \cdot d\left(\mathbf{r}, M_{1}(\lambda)\right)^{2} \quad \text { for all } \quad \mathbf{r} \in \mathscr{M} \\
& \text { with } \quad d\left(\mathbf{r}, M_{1}(\lambda)\right)<\epsilon .
\end{aligned}
$$

Proof: The functional $P_{\lambda}$ is bounded from below on $\mathscr{M}$ and can be shown to attain its infimum at some element $\hat{\mathbf{r}} \in \mathscr{M}$. Then $\hat{\mathbf{r}}$ is a stationary point of $P_{\lambda}$ on $\mathscr{H}$. From (2.12) it follows that $\hat{\mathbf{r}} \in M_{1}(\lambda)$, and thus that every element of $M_{1}(\lambda)$ minimizes $P_{\lambda}$ on $\mathscr{M}$.

In order to prove the estimate (3.15), let $\mathrm{r} \in \Sigma$ be given, and write $\mathrm{r}$ as in (3.14). The second variation of $P_{\lambda}$ on $\mathscr{M}$ at $\mathbf{r}_{1}=R(\psi) \hat{\mathbf{r}}_{1}$ is given by

$$
\begin{aligned}
J_{\lambda}^{1}(\boldsymbol{\xi}) & =\int_{0}^{2 \pi}\left\{\frac{1}{2} \sigma_{1}(\lambda) \boldsymbol{\xi}_{s} \cdot \boldsymbol{\xi}_{s}+\frac{1}{2} \lambda \boldsymbol{\xi}_{s} \cdot(\xi \times \mathrm{e})\right\} \mathrm{d} s= \\
& =\frac{1}{2} \lambda \int_{0}^{2 \pi}\left\{\boldsymbol{\xi}_{s} \cdot \boldsymbol{\xi}_{s}+\boldsymbol{\xi}_{s} \cdot(\boldsymbol{\xi} \times \mathrm{e})\right\} \mathrm{d} s
\end{aligned}
$$

for

$$
\boldsymbol{\xi} \in T \mathscr{M}\left(\mathbf{r}_{1}\right)=\left\{\boldsymbol{\xi} \in \Sigma \mid \xi_{s} \cdot\left(\mathbf{r}_{1}\right)_{s}=0\right\} .
$$

According to the lemma, part (ii), it follows that $J_{\lambda}^{1}(\xi)=0$ for all $\boldsymbol{\xi} \in T \mathscr{M}\left(\mathbf{r}_{1}\right) \cap S$. A simple calculation shows that, with $\mathbf{r}_{1}=R(\psi) \hat{\mathbf{r}}_{\mathbf{1}}$, $T \mathscr{M}\left(\mathbf{r}_{1}\right) \cap S=\left\{a \dot{R}(\psi) \hat{\mathbf{r}}_{1} \mid a \in \mathbb{R}\right\}$. Hence, if we restrict ourselves to functions $\xi \in T \mathscr{M}\left(\mathbf{r}_{1}\right)$ which satisfy the constraint in (3.14), then (3.5) implies:

$$
\begin{aligned}
& J_{\lambda}^{1}(\boldsymbol{\xi}) \geqslant c_{2}\|\xi\|_{1}^{2}, \quad \text { for all } \boldsymbol{\xi} \in T \mathscr{M}\left(\mathbf{r}_{1}\right), \\
& \text { with }\left(\boldsymbol{\xi}, \dot{R}(\psi) \hat{\mathbf{r}}_{1}\right)_{1}=0 .
\end{aligned}
$$

As in Theorem 1, this property of the second variation implies the desired result:

$$
P_{\lambda}(\mathbf{r})-P_{\lambda}\left(R(\psi) \hat{\mathbf{r}}_{1}\right) \geqslant \frac{1}{2}, c_{2}\left\|\mathbf{r}-R(\psi) \hat{\mathbf{r}}_{1}\right\|_{1}^{2}=\frac{1}{2} c_{2} d\left(\mathbf{r}, M_{1}(\lambda)\right)^{2}
$$

for $d\left(\mathbf{r}, M_{1}(\lambda)\right)$ sufficiently small.

\section{Theorem 4}

For $n \geqslant 2$, and $\lambda \geqslant \lambda_{n}$ the functional $P_{\lambda}$ has not a local minimum on $\mathscr{H}$ at the elements of the invariant sets $M_{n}(\lambda)$ : the difference $P_{\lambda}(\mathbf{r})-P_{\lambda}^{n}$ is not sign definite on $\mathscr{M}$.

Proof: Take any $\mathbf{r}_{n} \in M_{n}(\lambda)(n \geqslant 2)$. The second variation of $P_{\lambda}$ on $\mathscr{M}$ at $\mathbf{r}_{n}$ is given by

$$
J_{\lambda}^{n}(\boldsymbol{\xi})=\int_{0}^{2 \pi}\left\{\frac{1}{2} \sigma_{n}(\lambda) \boldsymbol{\xi}_{s} \cdot \boldsymbol{\xi}_{s}+\frac{1}{2} \lambda \boldsymbol{\xi}_{s} \cdot(\boldsymbol{\xi} \times \mathrm{e})\right\} \mathrm{d} s=
$$


60

$$
=\frac{1}{2} \cdot \frac{\lambda}{n} \int_{0}^{2 \pi}\left\{\xi_{s} \cdot \xi_{s}+n \xi_{s} \cdot(\xi \times \mathbf{e})\right\} \mathrm{d} s,
$$

for

$$
\xi \in T \mathscr{M}\left(\mathbf{r}_{n}\right)=\left\{\boldsymbol{\xi} \in \Sigma \mid \xi_{s} \cdot\left(\mathbf{r}_{n}\right)_{s}=0\right\} .
$$

We shall show that $J_{\lambda}^{n}$ can take negative values on $T \mathscr{M}\left(\mathbf{r}_{n}\right)$, which will prove the desired result. In fact, we shall show somewhat more, namely that this is true even if we restrict ourselves to functions $\xi \in T \mathscr{M}\left(\mathbf{r}_{n}\right) \cap \Sigma_{0}$, where $\Sigma_{0}=\{\boldsymbol{\xi} \in \Sigma \mid \boldsymbol{\xi}(2 \pi) \cdot \mathrm{e}=0\}$. Let $\Gamma_{\lambda}^{n}$ be the lowest eigenvalue corresponding to $J_{\lambda}^{n}$ on $T \mathscr{M}\left(\mathbf{r}_{n}\right)$ :

$$
\Gamma_{\lambda}^{n}:=\min \left\{J_{\lambda}^{n}(\boldsymbol{\xi}) \mid \xi \in T \mathscr{M}\left(\mathbf{r}_{n}\right) ;\|\xi\|^{2}=1\right\},
$$

and let

$$
\hat{\Gamma}_{\lambda}^{n}:=\min \left\{J_{\lambda}^{n}(\boldsymbol{\xi}) \mid \xi \in T \mathscr{M}\left(\mathbf{r}_{n}\right) \cap \Sigma_{0} ;\|\xi\|^{2}=1\right\} .
$$

Then $\Gamma_{\lambda}^{n} \leqslant \hat{\Gamma}_{\lambda}^{n}$. The main difficulty to prove that $\hat{\Gamma}_{\lambda}^{n}$ is negative, stems from the fact that the linearized constraint: $\xi_{s} \cdot\left(\mathrm{r}_{n}\right)_{s}=0$ explicitly depends on $s$.

The most elegant way to overcome this difficulty is to introduce local (Frenet) coordinate systems associated with $\mathbf{r}_{n}$. Define the tangent $\boldsymbol{\tau}=\left(\mathbf{r}_{n}\right)_{s}$, the principal normal $\mathbf{n}=\boldsymbol{T}_{s} \cdot\left(\boldsymbol{\tau}_{s} \cdot \boldsymbol{T}_{s}\right)^{-1 / 2}$, and the binormal $\mathbf{b}=\mathbf{\tau} \times \mathbf{n}$, and write $\boldsymbol{\xi}=\alpha \boldsymbol{\tau}+\beta \mathbf{n}+\gamma \mathbf{b}$. Then $\xi \in \Sigma_{0}$ if and only if $(\alpha, \beta, \gamma) \in \Sigma_{0}$. In terms of these functions $\alpha, \beta$ and $\gamma$, the number $\hat{\Gamma}_{\lambda}^{n}$ is given by

$$
\begin{aligned}
\hat{\Gamma}_{\lambda}^{n}= & \min \left\{\frac { \lambda } { 2 n } \int _ { 0 } ^ { 2 \pi } \left\{\alpha_{s}^{2}+\beta_{s}^{2}+\gamma_{s}^{2}+2 n\left[\alpha \beta_{s} \sin \theta(\lambda)+\right.\right.\right. \\
+ & \left.\left.\beta \gamma_{s} \cos \theta(\lambda)\right]\right\} \mathrm{d} s \mid(\alpha, \beta, \gamma) \in \Sigma_{0} \\
& \left.\alpha_{s}=n \sin \theta(\lambda) \cdot \beta ; \int_{0}^{2 \pi}\left\{\alpha^{2}+\beta^{2}+\gamma^{2}\right\} \mathrm{d} s=1\right\}
\end{aligned}
$$

It is now immediate that $\hat{\Gamma}_{\lambda}^{n}<0$ by verifying that for any $k$, with $1 \leqslant k<n$, a suitable multiple of $\left(\alpha_{k}, \beta_{k}, \gamma_{k}\right)$ :

$$
\begin{aligned}
\alpha_{k} & =\frac{n}{k} \sin \theta(\lambda)(1-\cos k s) \\
\beta_{k} & =\sin k s \\
\gamma_{k} & =-\frac{n}{k} \cos \theta(\lambda)(1-\cos k s)
\end{aligned}
$$

satisfies the constraints and renders a negative value to the functional in (3.23). This completes the proof. 


\section{Stability of the stationary states}

Let us now return to the evolution equations (2.5)-(2.7). The dynamical system under consideration is a conservative one: the total energy defined by

$$
E_{\lambda}(\mathbf{r}):=K\left(\mathbf{r}_{t}\right)+P_{\lambda}(\mathbf{r})
$$

is conserved during the motion:

$$
\frac{\mathrm{d}}{\mathrm{d} t} E_{\lambda}(\mathbf{r})=0 \quad \text { for solutions of }(2.1),(2.5)-(2.7)
$$

Let || $\mid \|_{1}$ be the norm defined by

$$
\|\mathbf{r}\|_{1}:=\left\|\mathbf{r}_{t}\right\|+\|\mathbf{r}\|_{1} .
$$

One uses in particular the following estimate:

$$
|\mathbf{r}(2 \pi, t)|^{2}=\left(\int_{0}^{2 \pi} \mathbf{r}_{s}(s, t) \mathrm{d} s\right)^{2} \leqslant 2 \pi \cdot\left\|\mathbf{r}_{s}\right\|^{2},
$$

valid for all functions $\mathrm{r}(s, t)$ with $\mathbf{r}(\cdot, t) \in \Sigma$, in order to show that $E_{\lambda}$ satisfies for some constant $C>0$ the estimate

$$
E_{\lambda}\left(\mathrm{r}^{1}\right)-E_{\lambda}\left(\mathrm{r}^{2}\right) \leqslant C \cdot\left\|\mathbf{r}^{1}-\mathrm{r}^{2}\right\|_{1}^{2}
$$

for all functions $\mathbf{r}^{i}(s, t), i=1,2$, for which $\mathbf{r}^{i}(\cdot, t) \in \Sigma$ for all $t$. With these preliminaries we can prove stability of those stationary states that have least potential energy (see e.g. Zubov [7] for definitions concerning Lyapunov stability).

\section{Theorem 5}

(i) For $0<\lambda<\lambda_{1}$ the stationary state $\mathbf{r}_{0}$ is stable in the sense of Lyapunov with respect to the norm $\||| \mid\|_{1}$.

(ii) For $\lambda>\lambda_{1}$ the invariant set $M_{1}(\lambda)$ is stable in the sense of Lyapunov with respect to the metric $\rho$, where $\rho$ is defined by:

$$
\rho\left(\mathbf{r}, M_{1}(\lambda)\right):=\left\|\mathbf{r}_{t}\right\|+d\left(\mathbf{r}, M_{1}(\lambda)\right) .
$$

Proof: (i) We show that the functional

$$
V_{\lambda}^{0}(\mathrm{r}):=E_{\lambda}(\mathrm{r})-E_{\lambda}\left(\mathrm{r}_{0}\right)=E_{\lambda}(\mathrm{r})
$$

is a Lyapnuov functional for the stationary state $\mathbf{r}_{0}$. Indeed, with Theorem 1 and the estimate (4.4), $V_{\lambda}^{0}$ satisfies for some constants $c>0$ and $\epsilon>0$ :

$$
c\|\| \mathbf{r}-\mathrm{r}_{0}\left\|_{1}^{2} \leqslant V_{\lambda}^{0}(\mathbf{r}) \leqslant C\right\|\left\|\mathbf{r}-\mathrm{r}_{0}\right\|_{1}^{2}
$$

for all functions $\mathbf{r}(s, t)$ for which $\mathbf{r}(\cdot, t) \in \mathscr{M}$ for all $t$ and $\left\|\mathbf{r}-\mathbf{r}_{0}\right\|_{1}<\epsilon$. As $V_{\lambda}^{0}$ is a constant of the motion because of (4.2), $V_{\lambda}^{0}$ is a Lyapunov functional, 
which proves the stability of $\mathbf{r}_{0}$.

(ii) Consider the functional

$$
V_{\lambda}^{1}(\mathbf{r}):=E_{\lambda}(\mathbf{r})-P_{\lambda}^{1}
$$

With Theorem 3 and (4.4) it follows that this functional satisfies for some constants $c>0$ and $\epsilon>0$ the estimates

$$
c \rho\left(\mathrm{r}, M_{1}(\lambda)\right)^{2} \leqslant V_{\lambda}^{1}(\mathrm{r}) \leqslant C \rho\left(\mathrm{r}, M_{1}(\lambda)\right)^{2}
$$

for all functions $\mathbf{r}(s, t)$ for which $\mathbf{r}(\cdot, t) \in \mathscr{M}$ for all $t$ and $\rho\left(\mathbf{r}, M_{1}(\lambda)\right)<\epsilon$. Together with (4.2) this implies that $V_{\lambda}^{1}$ is a Lyapunov functional for the invariant set $M_{1}(\lambda)$, which implies part (ii) of the theorem.

We use the first method of Lyapunov in order to show that the remaining stationary states are linearly unstable.

To that end, consider for $n=0$ and $\lambda>\lambda_{1}$ and for $n \geqslant 2$ and $\lambda>\lambda_{n}$ the equations (2.1), (2.5)-(2.7) linearized at $\mathbf{r}=\mathbf{r}_{n}, \sigma=\sigma_{n}(\lambda)$. Writing $\mathbf{r}=\mathbf{r}_{n}+\boldsymbol{\xi}$, $\sigma=\sigma_{n}(\lambda)+\nu$ in first order, there results

$$
\begin{aligned}
& \boldsymbol{\xi}_{t t}=\sigma_{n}(\lambda) \xi_{s s}+\lambda \boldsymbol{\xi}_{s} \times \mathrm{e}+\left(\nu\left(\mathbf{r}_{n}\right)_{s}\right)_{s} \\
& \left(\mathbf{r}_{n}\right)_{s} \cdot \boldsymbol{\xi}_{s}=0 \\
& {\left[\sigma_{n}(\lambda) \xi_{s}(2 \pi, t)+\nu(2 \pi, t)\left(\mathbf{r}_{n}\right)_{s}(2 \pi, t)\right] \cdot \mathrm{e}=0} \\
& \xi(0, t)=0, \xi(2 \pi, t) \times \mathrm{e}=0
\end{aligned}
$$

In order to show that these equations admit exponentially growing solutions, consider for $n=0$ and $n \geqslant 2$ the numbers $\Gamma_{\lambda}^{n}$ defined by (3.12) and (3.21). If $\xi=\xi^{n}(s)$ is a function that solves the minimization problem that defines $\Gamma_{\lambda}^{n}$, $\xi^{n}$ satisfies for some multiplier $\nu=\nu^{n}(s)$ the following set of equations:

$$
\begin{aligned}
& -\Gamma_{\lambda}^{n} \xi^{n}=\sigma_{n}(\lambda) \xi_{s s}^{n}+\lambda \xi_{s}^{n} \times \mathrm{e}+\left(\nu^{n}\left(\mathbf{r}_{n}\right)_{s}\right)_{s} \\
& \left(\mathbf{r}_{n}\right)_{s} \cdot \xi_{s}^{n}=0 \\
& {\left[\sigma_{n}(\lambda) \xi_{s}^{n}(2 \pi, t)+\nu^{n}(2 \pi, t)\left(\mathbf{r}_{n}\right)_{s}(2 \pi, t)\right] \cdot \mathrm{e}=0} \\
& \xi^{n}(0, t)=0, \xi^{n}(2 \pi, t) \times \mathrm{e}=\mathbf{0}
\end{aligned}
$$

Consequently, the functions:

$$
\begin{aligned}
\xi(s, t) & :=\xi^{n}(s) \exp \left[+t\left(-\Gamma_{\lambda}^{n}\right)^{1 / 2}\right] \\
\nu(s, t) & :=\nu^{n}(s) \exp \left[+t\left(-\Gamma_{\lambda}^{n}\right)^{1 / 2}\right]
\end{aligned}
$$

satisfy the equations (4.6). As $\Gamma_{\lambda}^{n}$ is negative in the interesting cases, we arrive at the following theorem.

\section{Theorem 6}

For $n=0$ and $\lambda>\lambda_{1}$, and for $n \geqslant 2$ and $\lambda>\lambda_{n}$, the stationary state $\mathbf{r}_{n}$ is 
linearly unstable: (4.8) are exponentially growing solutions of the linearized equations (4.6).

\section{The elastic string model}

In this final section we consider an elastic, completely flexible string whose ends are fixed at the origin and at the point $L \mathrm{e}=(L, 0,0)$ of a Cartesian coordinate system. Through the string an electric current of constant magnitude $J$ is sent, and the configuration is placed in a constant external magnetic field $B \mathrm{e}$, where the parameter $\lambda:=J B$ can take positive values.

The total mass of the string is normalized to be $2 \pi$, and we use a material (i.e. Lagrangian) variable $\mu, 0 \leqslant \mu \leqslant 2 \pi$, to describe a point at the string with the vector function $\mathbf{r}=\mathbf{r}(\mu, t)=(x(\mu, t), y(\mu, t), z(\mu, t)) ; \mu$ is the total mass of the string between the origin and the point under consideration. The potential energy per unit mass of such an elastic string is then described by a function $\mathscr{E}(l)$, where the specific length $l$ is defined by $l(\mu):=\mathrm{d} s / \mathrm{d} \mu$, with $s=s(\mu)$ the arc length.

The dynamical equations for this system can be derived with Hamilton's principle from the Lagrangian

$$
L\left(\mathbf{r}, \mathbf{r}_{t}\right)=\int_{0}^{2 \pi} \frac{1}{2} \mathbf{r}_{t} \cdot \mathbf{r}_{t} \mathrm{~d} \mu-P_{\lambda}(\mathbf{r})
$$

where now the total potential energy $P_{\lambda}$ is given by

$$
P_{\lambda}(\mathbf{r})=\int_{0}^{2 \pi}\left\{\mathscr{E}(l)+\frac{1}{2} \lambda \mathbf{r}_{\mu} \cdot(\mathbf{r} \times \mathrm{e})\right\} \mathrm{d} \mu
$$

We shall restrict ourselves to time-independent solutions, which are the stationary points of the functional (5.1).

For the following it is convenient to introduce the function $a^{2}(l):=$ $(1 / l)(\mathrm{d} \mathscr{E} / \mathrm{d} l)$, and we shall assume that

the function $l \rightarrow a^{2}(l)$ is positive, monotonically increasing for

$$
l \geqslant l_{0}:=L / 2 \pi
$$

in particular,

$$
a^{2}(l) \uparrow A^{2} \quad \text { for } \quad l \uparrow l^{*},
$$

where $A^{2}$ and $l^{*}$ may be finite or infinite.

Using the relation

$$
l^{2}=\mathbf{r}_{\mu} \cdot \mathbf{r}_{\mu},
$$

the equations for the stationary points of $P_{\lambda}$ are easily found to be

$$
\left(a^{2}(l) \mathbf{r}_{\mu}\right)_{\mu}+\lambda \mathbf{r}_{\mu} \times \mathrm{e}=\mathbf{0}
$$




$$
\mathrm{r}(0)=\mathbf{0}, \mathbf{r}(2 \pi)=L \mathrm{e}
$$

Taking the inner product of the equation in (5.5) with the tangent vector $\mathbf{r}_{\mu}$, it follows that $l$ must be a constant for any solution. With this observation, the solutions of (5.5) can be determined:

For any $\lambda>0$, the undeflected string configuration

$$
\mathbf{r}_{0}(\mu)=\left(l_{0} \mu, 0,0\right)
$$

is a solution with $l \equiv l_{0}$. Furthermore, for $n \in \mathbb{N}$, let $\lambda_{n}:=n a^{2}\left(l_{0}\right)$ and $\Lambda_{n}:=n A^{2}$, and denote rotation over an angle $\psi$ about the $x$-axis by $R(\psi)$ as before. Then, for $n \geqslant 1$ and $\lambda \in\left(\lambda_{n}, \Lambda_{n}\right)$ there are sets $M_{n}(\lambda)$ of solutions of (5.5), given by:

$$
\begin{aligned}
M_{n}(\lambda)= & \left\{\mathrm{r}=R(\psi) \hat{r}_{n} \mid \hat{\mathbf{r}}_{n}=l_{n}(\lambda)\left(\cos \theta(\lambda) \cdot \mu, \frac{1}{n} \sin \theta(\lambda) \cdot\right.\right. \\
& \left.\cdot \sin n \mu, \frac{1}{n} \sin \theta(\lambda)(1-\cos n \mu)\right), \\
& \left.l_{n}(\lambda) \cos \theta(\lambda)=l_{0} ; a^{2}\left(l_{n}(\lambda)\right)=\lambda / n ; 0 \leqslant \psi<2 \pi\right\} .
\end{aligned}
$$

[Note that for these time-independent solutions, we could just as well have used the $x$-coordinate as independent variable instead of $\mu$, as $x=l_{0} \mu$ for these solutions. For time-dependent solutions, however, $\mu$ is a more appropriate variable.]

Again, $\mathbf{r}_{n} \in M_{n}(\lambda)$ is a circle screw, lying on a cylinder of radius $R_{n}(\lambda)=$ $(1 / n)\left\{l_{n}^{2}(\lambda)-l_{0}^{2}\right\}^{1 / 2}$; because of the monotonicity assumption (5.2) for the function $a^{2}, R_{n}$ is a monotonically increasing function of $\lambda$. Determined by qualitative properties of the given function $a^{2}(l)$, three cases can be distinguished of the behaviour of the solutions $r_{n}$ as function of the parameter $\lambda$ :

(i) If $A^{2}=\infty$ and $l^{*}=\infty$, then $\Lambda_{n}=\infty$ and all solutions $\mathbf{r}_{n}, n \geqslant 1$, blow up' for $\lambda \rightarrow \infty: R_{n}(\lambda) \rightarrow \infty$ for $\lambda \rightarrow \infty$.

(ii) If $A^{2}=\infty$ and $l^{*}<\infty$, then $\Lambda_{n}=\infty$ and all solutions $\mathrm{r}_{n}, n \geqslant 1$ have bounded radius for $\lambda \rightarrow \infty: R_{n}(\lambda) \uparrow(1 / n)\left\{l^{* 2}-l_{0}^{2}\right\}^{1 / 2}$ for $\lambda \rightarrow \infty$.

(iii) If $A^{2}<\infty$ and $l^{*}=\infty$, then $\Lambda_{n}=n A^{2}$, and any solution $r_{n}, n \geqslant 1$, 'blows up' for a finite value of $\lambda: R_{n}(\lambda) \rightarrow \infty$ for $\lambda \uparrow \Lambda_{n}$.

As a specific example for which case (iii) occurs, consider an ideally elastic string, stretched between the fixed endpoints. The potential energy function $\mathscr{E}(l)$ then is given by

$$
\mathscr{E}(l)=\mathscr{E}_{0}+\sigma_{0}\left(l-l_{0}\right)+\frac{1}{2} Y\left(l-l_{0}\right)^{2},
$$

where $\mathscr{E}_{0}, \sigma_{0}$ and $Y$ are positive constants, the energy and stress at $l=l_{0}$ and Young's modulus respectively. The function $a^{2}(l)=Y\left(1-l_{0} / l\right)+\sigma_{0} / l$ satisfies the monotonicity condition (5.2), and $A^{2}=Y$. 
As has already been remarked, with the monotonicity condition (5.2), the bifurcation branches $R_{n}(\lambda)$ are monotonically increasing with $\lambda$. Note, however, that by relaxing the conditions on the function $a^{2}(l)$, the simple electromechanical system under consideration can serve to demonstrate a much more general behaviour of the bifurcation branches.

\section{Acknowledgement}

It is a pleasure to have the opportunity to thank Dr. H.J. van Ouwerkerk for his assistance during the preparation of [4]. His lasting interest in elucidating the physics in this, and many other, string models, has been very helpful and is appreciated above all.

\section{References}

1. Broer LJF (1970) On the dynamics of strings. J Eng Math 4: 195-202

2. Broer LJF (1970) Longitudinal Motion of an Elastic Bar. J Eng Math 4: 1-8

3. Broer LJF and van Groesen EWC (1977) Simple wave interaction of an elastic string. Rheol Acta 16: 161 168

4. van Groesen EWC (1973) Stroomvoerende draad in magneetveld: Stabiliteit van de stationaire toestanden. Master thesis, Eindhoven University of Technology

5. van Groesen EWC (1978) Variational Methods in Mathematical Physics. Thesis, Eindhoven University of Technology

6. Ioffe AD and Tihomirov VM (1979) Theory of Extremal Problems. Amsterdam: North-Holland

7. Zubov VI (1964) Methods of A.M. Lyapunov and their applications. Groningen: Noordhoff 